\title{
Indicadores tempranos de desarrollo pragmático y alteraciones del vínculo de apego
}

\section{Early Indicators of Pragmatic Development and Attachment Disorders}

\author{
Iria Botana, Manuel Peralbo y Manuel García-Fernández \\ Departamento de Psicología. Universidade da Coruña,España.
}

\begin{abstract}
Resumen
El objeto de este trabajo es analizar el impacto de los hitos pragmáticos del desarrollo infantil en la detección precoz de los trastornos de la relación entre niños y cuidadores. La relevancia del trabajo reside en el valor predictivo de los marcadores de desarrollo pragmático temprano y su papel dentro de la díada madre-hijo. El estudio emplea los datos extraídos durante el proceso de baremación de la escala EDPRA. Los resultados sugieren que los Items de base interactiva que requieren de un adulto disponible se pueden asumir como indicadores tempranos de un apego seguro. La conclusión obtenida apunta al perfil de desarrollo pragmático del niño como signo de alerta del tipo de vínculo establecido con su cuidador principal

Palabras clave: Indicadores temprano, alteraciones del apego, desarrollo pragmático
\end{abstract}

\begin{abstract}
The purpose of this paper is to analyze the impact of the pragmatic milestones of child development in the early detection of disorders of the relationship between children and caregivers. The relevance of the study lies in the predictive value of early pragmatic development markers and their role within the mother-child dyad. The study uses the data extracted during the EDPRA scale construction process. The results suggest that the interactive base items that require an available adult can be assumed as early indicators of a secure attachment. The conclusion obtained points to the pragmatic development profile of the child as an alert sign of the type of bond established with its main caregiver

Keywords: Early indicators, Attachment Disorders, Pragmatic Development
\end{abstract}

\section{Introducción}

Uno de los grandes logros de los investigadores de lo humano, ha sido demostrar la importancia de las relaciones afectivas y de los cuidados mutuos en la conservación de la salud psíquica, tal y como recoge Barudy (2005). De hecho diversos estudios (Bowlby, 1976), señalan la presencia de graves problemas en el desarrollo integral de los niños y las niñas, ante la ausencia de una relación afectiva temprana, eficaz, es decir, la falta de una base segura para el desarrollo.

La teoría del apego enfatiza el rol crucial y formativo que desempeñan las experiencias tempranas, especialmente te durante los dos primeros años de vida, en el desarrollo social y emocional del ser humano. A través de interacciones repetidas con las figuras de apego los niños forman modelos operativos (Anglin, Cohen y Chen, 2008). Sabemos que entre las conductas maternas que promueven un apego seguro se encuentran la disponibilidad, la consistencia, la contingencia en las respuestas, la cooperación y el mantenimiento de un clima afectivo positivo para la interacción (Cassidy \& Shaver, 1999). Por lo tanto la comunicación y el lenguaje, mediadores principales en cuanto a consistencia, contingencia, disponibilidad, respuesta y cooperación, podrían actuar de signos de alerta ante la existencia o no de una base segura. Aun así, todavía no sabemos con exactitud cuáles son los marcadores comunicativos que evidencian una falta de apego seguro.

Los vínculos entre padres e hijos, se forman y se mantienen a partir de mecanismos biológicos, existiendo una fuerte relación entre las relaciones tempranas con los cuidadores y el desarrollo de las acciones y patrones comunicativos que inician los niños. Por lo tanto es esperable que la conducta interactiva entre el niño y su cuidador se manifieste de manera temprana a través de marcadores propiamente pragmáticos, puesto que la manifestación de la interacción es precursora de la manifestación del propio lenguaje. Esta relación posibilita el uso de los marcadores pragmáticos como indicadores tempranos en las alteraciones del vínculo entre madre-hijo

El objetivo de este trabajo es determinar si la escala EDPRA podría ser utilizado también como herramienta de cribado, no sólo para las alteraciones en el desarrollo pragmático, sino también para la identificación de riesgo potencial en otros trastornos del desarrollo.

\section{Metodología}

\section{Participantes}

El muestreo se realizó siguiendo un criterio de conveniencia. Como criterio de exclusión se descartaron aquellos casos en los que pudiera existir sospecha de una alteración que afecte al desarrollo comunicativo, lingüístico o cognitivo de los niños. Además, fueron considerados también criterios de exclusión la existencia de deficiencia auditiva moderada o grave, deficiencia visual severa, síndrome de filiación genética, grandes prematuros, trastorno del neurodesarrollo, trastornos del 
sistema nervioso central, hospitalización prolongada, institucionalización durante los primeros meses de vida.

La muestra se extrajo de la base de datos del Servicio de Atención Temprana (SAT). Tras una revisión de los casos entrantes en los últimos doce meses se seleccionaron los que previamente habían participado en la baremación de la escala EDPRA. Del grupo resultante de 19 niños y niñas se encontraron dos de ellos con idéntica hipótesis diagnóstica

\section{Instrumentos}

\section{Anamnesis}

Entrevista extensa con los padres en la cual se recogen los datos sobre en periodo prenatal y posnatal del menor así como la situación familiar durante estos períodos. También se hacen referencias a los hitos evolutivos a través de las descripciones de los padres y a las rutinas que tuvo y tiene el menor.

\section{Cuestionario para padres ASQ-3 (Squires y Bricker, 2009).}

El ASQ-3 está compuesto por 21 cuestionarios diseñados para ser respondidos por padres y cuidadores de niños entre 1 mes y 5 años y medio de edad. Cada cuestionario contiene 30 preguntas sobre las siguientes áreas de desarrollo:

Comunicación: mide las habilidades verbales de su hijo, que incluyen tanto la comprensión y la expresión

Motora Gruesa: explora cómo el niño utiliza los brazos, las piernas y otros músculos para sentarse, gatear, caminar, correr y para hacer otras actividades.

Motora Fina: mide aspectos tales como la coordinación ojos-manos y la manipulación de pequeños objetos.

Resolución de problemas: explora cómo el niño soluciona problemas y juega.

Personal-Social: observa cómo el niño juega solo, con juguetes y/o con otros niños para explorar su capacidad de ayudarse a sí mismo y de interacción con las demás personas

\section{Situación Extraña (Ainsworth, Blehar, Waters y Wall, 1978).}

Esta tarea tiene por objetivo activar la manifestación de la conducta de apego a partir de la observación de la reacción del niño ante la situación extraña. Los creadores de la prueba describen distintos patrones de apego:

Apego Seguro: Los niños que presentan este tipo de apego, juegan y exploran cuando están con su madre, se disgustan cuando la madre se va y demandan el reencuentro. Cuando la madre vuelve se consuelan fácilmente y vuelven a jugar.

Apego Inseguro-Evitativo: Los niños que muestran estra patrón de apego evitan la proximidad a la madre, no muestran signos de disgusto cuando ésta se va y cuando vuelve no muestran conductas de acercamiento a ella. Apego Inseguro-ambivalente: Los niños reaccionan fuertemente a la marcha de la madre; cuando la madre regresa pueden buscarla o mostrar rabia o pasividad, no se consuelan fácilmente y no retoman la actividad exploratoria previa a la separación.

En esta prueba también se evalúa el estilo de conducta de la madre. La madre de un niño con apego seguro se muestra sensible a las señales del bebé, las interpreta adecuadamente y responde de manera apropiada.

Las madres de niños con un patrón de conducta inseguroevitativo le transmite al niño de forma involuntaria que sus señales no son efectivas, no son funcionales y no obtendrán la respuesta esperada.

Por otro lado las madres de los niños con un patrón de apego inseguro-ambivalente son inconsistentes, cambiantes en sus respuestas, lo que hace que el niño tenga modelos contradictorios al no poder predecir la conducta de la madre.

\section{Merrill-Palmer-Revised Scales of Development (Roid y Sampers, 2004)}

Las escalas de desarrollo Merrill-Palmer-R fueron desarrolladas para la evaluación de las cinco áreas principales del desarrollo; cognición, lenguaje, motricidad, conducta adaptativa y autocuidado y socioemocional. Ofrece información sobre el desarrollo global del niño para valorar la presencia de posibles retrasos en alguna de las áreas

\section{Escala de Desarrollo Pragmático (EDPRA).}

La escala EDPRA cuenta con tres ejes de evaluación pragmática: Funciones comunicativas, respuesta a la comunicación e interacción y conversación. Cada eje incluye un máximo de 6 ítems puntuables de 0-3 (Botana y Peralbo, 2015). Dispone de una hoja de respuesta con sus correspondientes ítems para cada rango de edad: 6 a 12 meses, 12 a 24 meses, 24 a 36 meses y 36 a 48 meses.

\section{Procedimiento}

En primer lugar se realizó un cribado entre los usuarios del servicio de Atención Temprana, de un total de 110 usuarios activos en ese momento se extrajeron los datos de aquellos que previamente habían participado en el proceso de baremación de la escala EDPRA. Formando estos un total de 19 niños y niñas. De esta muestra, dos niños, ambos de 10 meses de edad en el momento de la aplicación de la EDPRA, obtuvieron puntuaciones inferiores a los niños de su edad en 5 de los 18 items aplicados. Transcurrido un tiempo, 9 y 10 meses después respectivamente, ambos niños demandan atención temprana en su centro de referencia. Se les aplicó la ASQ-3, la Situación Extraña, y las escalas de desarrollo Merrill-Palmer-R.

\section{Resultados}

Tras analizar los expedientes de ambos niños y las pruebas administradas en el SAT se encuentran coincidencias en la valoración de ambos y en sus hipótesis diagnósticas.

-Anamnesis: en ambas historias clínicas se recogen coincidencias en los tiempos dedicados por las madres. Ambas madres con nivel socioeducativo medio-alto que por motivos diferentes (una por enfermedad del padre y otra por trabajo) deben ausentarse durante pequeños 
períodos de tiempo con escasa disponibilidad, durante los primeros 12 meses de vida del niño y con grandes cargas a nivel de estrés.

-ASQ-3: En los dos casos se obtienen `puntuaciones bajas en el área de resolución de problemas y personalsocial

-Escalas de desarrollo Merrill-Palmer-R: ambos casos obtienen puntuaciones bajas en el área socioemocional, y medio bajos en lenguaje y cognitiva, condicionadas por tiempos de atención acortados.

-Situación extraña: los niños no buscan la proximidad a sus cuidadoras principales, no muestran signos de disgusto cuando ésta se van y cuando vuelven no muestran conductas de mantenimiento del contacto hacia ellas. Ambas madres modifican la respuesta a las conductas de sus hijos de forma rápida e inconsistente.

Tras la valoración por el equipo de atención temprana de las pruebas administradas y la observación clínica llevada a cabo en las sesiones de evaluación se elabora un informe inicial en el que consta como hipótesis diagnóstica inicial: Alteración en la interacción social y en la atención asociado a un posible apego inseguroevitativo

El análisis de los resultados obtenidos previamente por los dos niños en el proceso de baremación de la escala EDPRA constata que ambos obtuvieron puntuaciones inferiores a sus iguales en edad en 5 items de la escala para 6 a 12 meses EDPRA:

Tabla 1.

Items de la escala EDPRA con puntuaciones inferiores a las esperadas.

\begin{tabular}{ll}
\hline Item & \\
\hline- & Señala con función de petición \\
- & Realiza interacciones básicas mediante: \\
& Saludo-despedida / Tira un beso si se lo \\
piden/ofrece un juguete al adulto & \\
- & Solicita atención, objetos o información. \\
- & $\begin{array}{l}\text { Responde a órdenes dadas por el adulto } \\
\text { apoyadas con gestos } \\
\text { - }\end{array}$ \\
\end{tabular}

Tras realizar un análisis de los items, se encuentra una posible relación entre pragmática y apego: la necesidad de un adulto disponible. Cada uno de los ítems en los que estos dos niños recibían puntuaciones inferiores a sus compañeros de 10 meses dependían directamente de la disponibilidad del adulto de referencia, alguien a quien dirigir todas y cada una de las acciones iniciadas por el niño. Ese adulto es el que puede proporcionar la disponibilidad, la consistencia, la contingencia en las respuestas y mantener un clima afectivo positivo para la interacción.

\section{Discusión y Conclusiones}

Los hitos evolutivos recogidos en estos cinco items se relacionan fuertemente con el concepto de intersubjetividad tal y como lo recogía Stern (1991), que posteriormente dará paso a la comunicación con el otro, con quien el niño interactúa porque sabe que obtendrá una respuesta consecuente a su acción. Ello le permite reafirmar su acción y empezar a compartir el foco de atención, las intenciones, los estados afectivos. También los enfoques más cognitivos y/o lingüísticos recogen la disponibilidad del adulto como un predecesor de la comunicación. Bruner (1975) entre otros, habla de distintos hitos evolutivos como la búsqueda mediante señalado, juegos de toma y dame como interacciones básicas que en última instancia conducen al descubrimiento de la intersubjetividad. En los items analizados el peso de la acción del adulto es muy significativo. El adulto interpreta las conductas del niño, es decir, le atribuye un significado. Durante este proceso las variaciones en la respuesta o la inexistencia de la misma, modificarán el patrón de conducta del niño.

El desarrollo de las acciones infantiles, que más adelante se convierten en formas de comunicación, no es mera consecuencia de la maduración. Seguramente su formación tiene como fin conseguir una comunicación con las personas que le rodean, mediante su asimilación. Tan solo con un contexto comunicativo disponible estos actos surgen en el niño y éste empieza a utilizarlos como formas de comunicación. Por lo tanto cada uno de estos actos dependerá en gran medida de la situación concreta en la que transcurre la intercomunicación niño-adulto. Cada una de las acciones evaluadas a través de los items de la escala, necesitan un entrenamiento y una generalización, es decir, un contexto en el que una acción puntual se convierta en un acto con contenido social e interactivo, que el niño repetirá en situaciones similares. Para ello es imprescindible que los formatos de interacción se repitan un número suficiente de veces de tal forma que el bebé llegue a anticipar la conducta del adulto de referencia ante su actuación. Esto no es posible en los casos en los que el adulto no se encuentra disponible, en los dos casos objeto de estudio las madres que inicialmente se encargaron de la crianza tuvieron situaciones que les impidieron continuar con actitud disponible durante el primer año de vida del bebe.

\section{Referencias}

Ainsworth, M. D., Blehar, M. C., Waters, E., \& Wall, S. (1978). Patterns of attachment: A psychological study of the strange situation. Hillsdale: LEA

Anglin, D. M., Cohen, P. R., \& Chen, H. (2008). Duration of early maternal separation and prediction of schizotypical symptoms from early adolescence to midlife. Schizophrenia Research, 103(1), 143-150. https://doi.org/10.1016/j.schres.2008.02.016

Barudy, J. \& Dantagnan, M. (2005). Los buenos tratos a la infancia: parentalidad, apego y resiliencia. Barcelona: Gedisa

Botana, I. \& Peralbo, M. (2015). Estudio sobre la adaptacion y validación de un instrumento de evaluación del desarrollo pragmático temprano [Edición especial] Revista de estudios e investigación en psicología y educación, 9, 39-42. https://doi.org/10.17979/reipe.2015.0.09.374.g641

Bowlby, J. (1976). Attachment and loss. Vol. 2: Separation: Anxiety and anger. New York: Basic books. 
Bruner, J. (1981). De la comunicación al lenguaje: una perspectiva psicológica. Infancia y Aprendizaje, 4, 133-143

Cassidy, J. (1999). The nature of the child's ties. En J. Cassidy y P. Shaver (Eds) (1999). Handbook of attachment: Theory, research, and clinical applications (pp. 3-20). New York: Guilford Press

Roid, G. H. \& Sampers, J. L. (2004). Merrill-Palmerrevised: scales of development. Illinois:Stoelting.

Stern, D. N. (1991). El mundo interpersonal del infante: una perspectiva desde el psicoanálisis y la psicología evolutiva. Buenos Aires: Paidos.

Squires, J. \& Bricker, D. (2009). Ages \& Stages

Questionnaires: A Parent-Completed ChildMonitoring System. Baltimore: Brookes Publishing Company. 